\title{
Investigación en pedagogía ciudadana 2008 - 2017
}

Research in citizen education 2008 - 2017
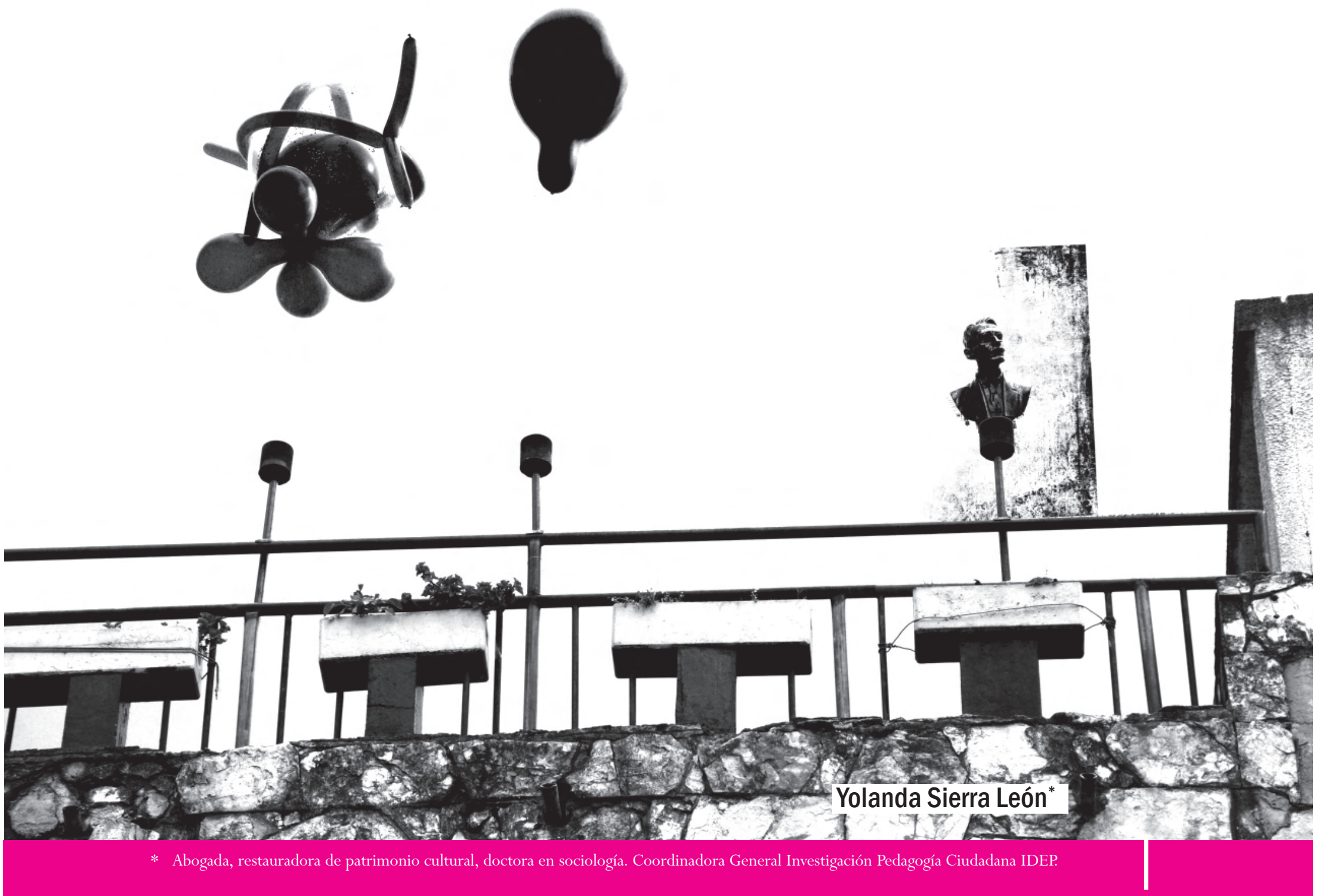
Resumen El Instituto para la Investigación Educativa y el Desarrollo Pedagógico IDEP, consciente de la importancia de fortalecer la Cultura Ciudadana en Bogotá, ha propuesto, en el marco del Plan de Desarrollo Bogotá Positiva, adelantar una investigación llamada "Pedagogía Ciudadana". Ésta es una investigación longitudinal-aplicada que busca diseñar, implementar y evaluar un proyecto pedagógico para la formación en cultura ciudadana de niños, niñas y jóvenes de Bogotá, así como implementar un sistema de medición en cultura ciudadana para esta población. El programa se desarrollará en ocho años, trabajando desde el 2008 al 2017, con niños, niñas y jóvenes escolarizados, de colegios oficiales y privados, de las 20 localidades de Bogotá. Incluye una muestra representativa de instituciones escolares: colegios grandes, medianos y pequeños; femeninos, masculinos y mixtos; laicos y religiosos; de calidad alta y baja; de diferentes estratos socioeconómicos y con diversos niveles de convivencia escolar.

Palabras claves: Derechos Colectivos, ciudad, ciudadanía, cultura, cultura ciudadana,
democracia, pedagogía y educación.

Abstract The Institute for Educational Research and Pedagogic Development (IDEP), being aware of the importance of strengthening civic culture in Bogota, has proposed under the frame Development Plan Bogotá Positiva, to carry a research called "Citizen Education". This is a longitudinal-applied research that seeks to design, implement and evaluate an educational project for the formation of children and adolescents in civic culture in Bogota, together with the implementation of a measurement system in civic culture for this population. The Program will be developed during eight years working from 2008 to 2017 with children and teenagers that are enrolled in public and private schools of the 20 boroughs of Bogotá. It is planned with a representative sample that includes large, medium and small schools; feminine, masculine or both;, secular and religious; high and low quality; of different socioeconomic strata; and with different levels of school-coexistence.

Keywords: Collective Rights, city, citizenship, culture, civic culture, democracy, pedagogic and education. 


\section{Presentación}

La cultura ciudadana puede ser entendida ampliamente como el resultado de un proceso social donde se construyen nociones sobre el deber ser en sociedad. Estas pautas pueden cambiar de un contexto social a otro, así como las maneras en las que se dan a conocer estas nociones entre los ciudadanos.

Desde esta perspectiva, la ciudad es educativa perece: las prácticas sociales, el territorio, los deportes, la manera como se transita, las cuestiones medioambientales y de salud; las económicas y presupuestarias; las que se refieren a la movilidad, a la seguridad, a los diferentes servicios; las correspondientes a los medios de comunicación, son formas de educación de la ciudadanía." "Desde este punto de vista, "la ciudad que educa" es un proyecto compartido que involucra a la administración pública y a la sociedad en general.

A este respecto, es importante señalar que en Bogotá existen varias experiencias sobre cultura ciudadana que son desarrolladas, en algunos casos, por las administraciones distritales, y en otros, por el sector

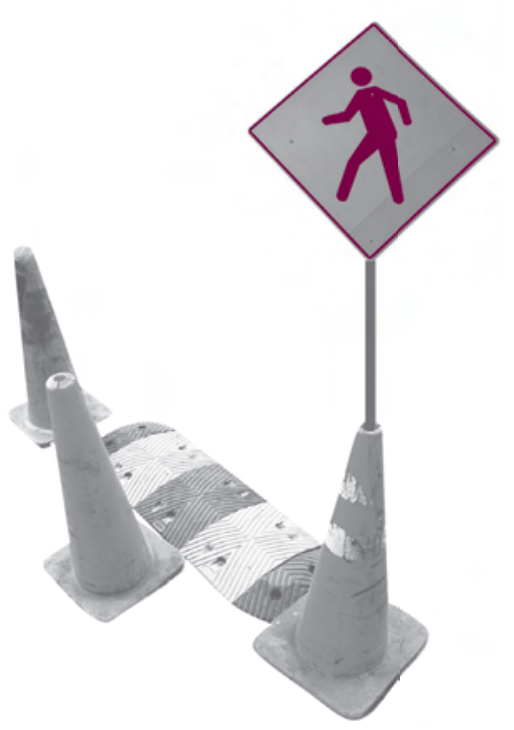

educativo, las cuales están focalizadas en trabajo con población escolar en el tratamiento de temas y desde perspectivas particulares de las instituciones educativas. En estas experiencias, la cultura ciudadana se identifica como una necesidad permanente, materializada su importancia en cada una de las mediciones, en los planes de desarrollo de las administraciones y en las múltiples experiencias dentro de las aulas.

No obstante, así hablar de cultura ciudadana sea un tema de permanente discusión, hoy en día, cuando hacemos un balance del tema, y además lo contrastamos con la situación actual, en términos de indicadores de convivencia, pertenencia, reconocimiento de derechos y deberes, respeto por el patrimonio público 
o participación, no queda muy claro cómo ha sido el proceso que la propia ciudad ha experimentado y ha realizado frente al tema. La observación de este proceso es difícil, ya que no existe un seguimiento de las intervenciones que permita identificar cuáles fueron los impactos que tales experiencias han tenido para la ciudad y cuál ha sido el efecto concreto de estas experiencias.

Una de las razones en las que radica la dificultad de observar el tema de cultura ciudadana como un proceso, es debido a que las acciones de mayor impacto -en términos de la población involucrada - se han dado en adultos, por lo que se ha hecho un énfasis en la transformación rápida de las acciones que se considera no están en concordancia con la cultura ciudadana. En este sentido, la ciudad no cuenta con un seguimiento a largo plazo mediante el cual se puedan identificar cuáles alternativas realmente promueven los cambios que se quieren obtener.

Adicionalmente, el objetivo de estas iniciativas gubernamentales en Bogotá, generalmente han estado orientados a lograr las metas que se proponen desde los planes de desarrollo, como es el caso de la transformación de indicadores sociales. Sin embargo, la cuestión radica en ¿cómo debe plantearse una iniciativa en cultura ciudadana que sobrepase los cambios administrativos y promueva la creación de cultura ciudadana a largo plazo?

En este punto se centra la investigación, pues se quiere atender a la necesidad de construir un proyecto en cultura ciudadana de largo alcance, monitoreado previamente antes de ser implementado y con un sistema de medición que contribuya a diseñar políticas públicas permanentes en la ciudad. En este sentido, se persigue un proyecto pedagógico que forme agentes sociales dentro de un espacio compartido como lo es la ciudad.
Así, en lugar de ver la cultura ciudadana como resultado, se pretende una estrategia sostenible, medible y observable a lo largo del tiempo, la cual permita, a futuro, identificar cuáles son las formas en las que se da la construcción de la ciudadanía desde los primeros procesos de formación en población infantil y juvenil.

Partiendo de esta posición, se desprenden nuevos interrogantes frente a un proceso de formación en cultura ciudadana, por ejemplo: ¿cuál es la estrategia pedagógica apropiada para formar en cultura ciudadana a la población infantil y joven de la ciudad?, ¿es diferente la metodología requerida para los niños con respecto a la de los adultos?, ¿es posible tener una misma estrategia pedagógica para poblaciones escolares con diferentes características sociales, económicas y culturales? ¿Cuál es el estado de la cultura ciudadana en niños, niñas y jóvenes de Bogotá, en diferentes momentos del período 2008-2017?

La investigación en Pedagogía Ciudadana del IDEP, para responder estos interrogantes sobre las estrategias pedagógicas, la metodología y el reconocimiento de las diferencias entre los escolares de Bogotá, propone el siguiente punto de partida, que constituye la filosofía central de la línea de intervención pedagógica: (Infra 2. v)

La formación cognitiva, afectiva y comportamental en Derechos Colectivos mejora los indicadores de Cultura Ciudadana.

El anterior punto de partida surge del concepto de la cultura ciudadana, del predominio concedido a los derechos colectivos, y de la convicción de que la formación requiere un proceso integral entre lo racional, lo afectivo y lo comportamental. 
De otra parte, y ante el interrogante sobre el estado de la cultura ciudadana de la población escolar, la investigación cuenta con la línea de observación (Infra III), la cual provee instrumentos de medición y evaluación, tanto de la cultura ciudadana en población escolar, como de los instrumentos pedagógicos utilizados en el programa.

\section{Derechos Colectivos, Cultura Ciudadana y Pedagogía}

La ciudad requiere, entonces, de procesos de formación a largo plazo en cultura ciudadana. Esta necesidad precisa de varios conceptos involucrados en el proceso pedagógico, tales como: ciudad, democracia, ciudadanía, cultura, cultura ciudadana, derechos colectivos, educación y pedagogía.

Ciudad: la vida en sociedad implica, entre otras cosas, la interacción entre sujetos dentro de un espacio determinado, en este sentido, la ciudad como aquel espacio compartido y la ciudadanía como la construcción de estos sujetos, se convierten en el primer punto de reflexión sobre la cultura ciudadana.

El programa Pedagogía Ciudadana reconoce el concepto de ciudad ofrecido por el urbanista J. Borja como el más pertinente para sus fines operativos. Así, este autor define la ciudad como "aquel producto físico, político y cultural complejo, europeo y mediterráneo, pero también americano y asiático, que hemos caracterizado en nuestra ideología y en nuestros valores como concentración de población y actividad, mezcla social

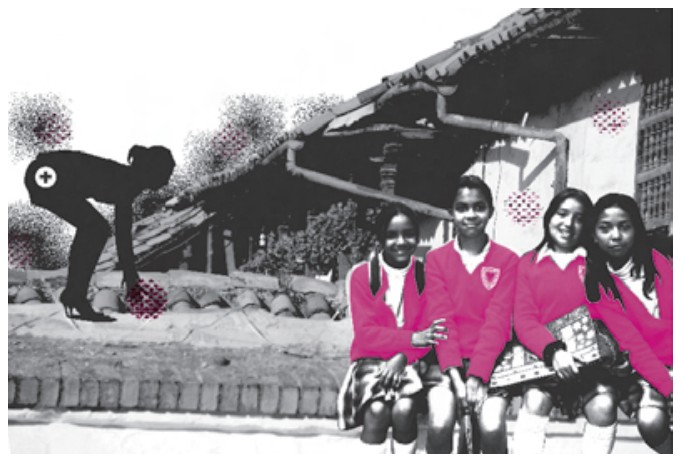

y funcional, capacidad de autogobierno y ámbito de identificación simbólica y de participación cívica. Ciudad como lugar de encuentro, de intercambio, ciudad igual a cultura y comercio. Ciudad de lugares y no un mero espacio de flujos". ${ }^{2}$

En este sentido, la ciudad, según la caracterización de Borja, es un espacio compartido gracias a que recoge dentro de su multidimensionalidad la acción propia de los seres humanos, tanto a nivel material, evidente en el espacio físico, como de organización política y cultural; de creación de representaciones y significados, con la creación de lugares, de historia colectiva y de pautas hacia el futuro. 
Democracia: caracterizar cultura ciudadana como un tema de reflexión implica, al tiempo, hablar de una manera de concebir la vida en sociedad. Por tanto, el programa Pedagogía Ciudadana parte de considerar la cultura ciudadana desde un principio democrático.

Desde esta perspectiva, la democracia no es entendida exclusivamente como marco legal de organización del estado colombiano. La democracia, siguiendo a Arendt ${ }^{3}$, evoca algo que tiene que ver con la propia condición de lo humano. En este sentido, la democracia implica la posibilidad que cada uno tiene de ser sí mismo, de interactuar con otros y de realizar sus acciones dentro de un modelo de igualdad y, a la vez, de diferencia ${ }^{4}$, de tal manera que cada uno pueda actuar partiendo de su particularidad, ya que no todos por el hecho de estar o de compartir un espacio somos idénticos, y además, no siempre todos partimos de una misma situación para llevar a cabo una acción.

Ciudadanía: el concepto de ciudadanía no es un concepto que tenga una definición universalmente aceptada. Éste es usualmente modificado en las prácticas políticas y acomodado a los cambios históricos. Tradicionalmente, la ciudadanía se ha entendido como la condición social de un habitante nacido o naturalizado en una ciudad o estado, que lo hacían acreedor a un conjunto de derechos y deberes civiles, sociales y políticos, que se podían ejercer en el ámbito del territorio de la ciudad ${ }^{5}$. Existen otras aproximaciones más contemporáneas al concepto, como la ofrecida por Cortina (1997): “... el estatuto de ciudadano es, en consecuencia, el reconocimiento oficial de la integración del individuo en la comunidad política, comunidad que desde los orígenes de la Modernidad cobra la forma del Estado nacional de derecho» ${ }^{6}$.

Este reconocimiento, dentro del sistema político colombiano, sólo se adquiere a la edad de 18 años. Sin embargo, el concepto ha evolucionado y, hoy en día, la definición moderna de ciudadanía abarca terrenos más amplios que la participación en la política formal, extendiéndose a campos como el cultural, medioambiental, educacional, entre otros, lo que significaría que la mayoría de edad para votar o para ocupar puestos públicos no es una limitante para el ejercicio de la ciudadanía en otros ámbitos.

La ciudadanía, como cualquier rol, requiere para que se construya su ejercicio de un proceso de socialización y de formación que supera en mucho el conocimiento de los derechos de los que se es titular, y supone la existencia de espacios que otorguen la oportunidad de constituirse en sujetos de derechos y responsabilidades en la práctica cotidiana. A este respecto, muchas teorías coinciden en definir los primeros años de vida como determinantes para la integración de los sujetos en las sociedades.

En otros términos, la construcción de ciudadanía implica el desarrollo de disposiciones, valores y creencias, lo que es tan importante para la acción colectiva como la organización de los sujetos. En la dimensión de la cultura ciudadana es importante desarrollar valores como la libertad, la igualdad, la solidaridad, la tolerancia, la convivencia y la cooperación, debido a que son valores que están relacionados con las vías en que han de desarrollarse las relaciones entre unos y otros.

\footnotetext{
3 Arent, Hanna. La condición Humana. España: Paidós, 1993.

4 Touraine, Alain. Igualdad y Diversidad. México: FCE, 1998.

5 Borja, J. La ciudad conquistada. Madrid: Alianza Editorial, 2003.

6 Cortina, A. Ciudadanos del mundo, hacia una teoría de la ciudadanía. Madrid: Alianza Editorial, 1997.
} 


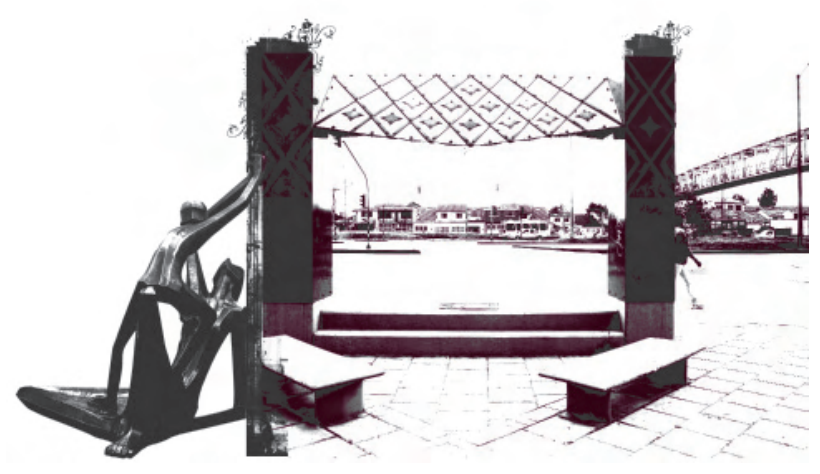

Cultura: la caracterización del concepto de cultura, como cualquier construcción histórica, requiere ser objetivada en el sentido de que cada noción de la misma implica la movilización de una concepción sobre lo que debe considerarse culturalmente legítimo y lo que no lo es.

En este sentido, para el programa Pedagogía Ciudadana, la cultura no es concebida exclusivamente desde el punto de vista antropológico, según el cual, cultura se refiere a toda manifestación de las maneras de ver, sentir y concebir el mundo. Para el programa la cultura también hace referencia a los procesos de construcción colectiva que se generan en un contexto social y cultural particular, que responden a procesos intencionales de búsqueda o transformación de prácticas y da cuenta de los procesos que algunos grupos pueden emprender en la búsqueda de objetivos colectivos.
En ciencias sociales son muchos los autores que se han aproximado al término cultura, razón por la que no se encuentra un consenso general en lo que respecta al término. Sin embargo, uno de los autores que proporciona una de las definiciones más ampliamente utilizadas es Edward Tylor (1909). Según este autor, la cultura es "aquel todo complejo que incluye el conocimiento, las creencias, el arte, la moral, el derecho, las costumbres, y cualesquiera otros hábitos y capacidades adquiridos por el hombre. La situación de la cultura en las diversas sociedades de la especie humana, en la medida en que puede ser investigada según principios generales, es un objeto apto para el estudio de las leyes del pensamiento y de la acción del hombre"7.

No obstante, autores más contemporáneos como Max Weber definen la cultura desde la siguiente perspectiva: “...el hombre es un animal inserto en tramas de significación que él mismo ha tejido, considero que la

7 Taylor, E. Anthropology: An Introduction to the Study of Man and Civilization. New York: D. Appleton, 1909. 
cultura es esa urdimbre y que el análisis de la cultura ha de ser por lo tanto, no una ciencia experimental en busca de leyes, sino una ciencia interpretativa en busca de significaciones". ${ }^{8}$

Así, el programa de Pedagogía Ciudadana entiende en la cultura la necesidad de identificar aquello que es común a un grupo de personas, ya sean los hábitos, las costumbres como nos propone Tylor, o las tramas de significación propuestas por Weber. Con estos elementos comunes podemos definir entonces que se entiende por cultura ciudadana.

Cultura ciudadana: la categoría de cultura ciudadana aparece por primera vez en el contexto de la ciudad de Bogotá, en el plan de gobierno del Alcalde Mockus, como "el conjunto de costumbres, acciones y reglas mínimas

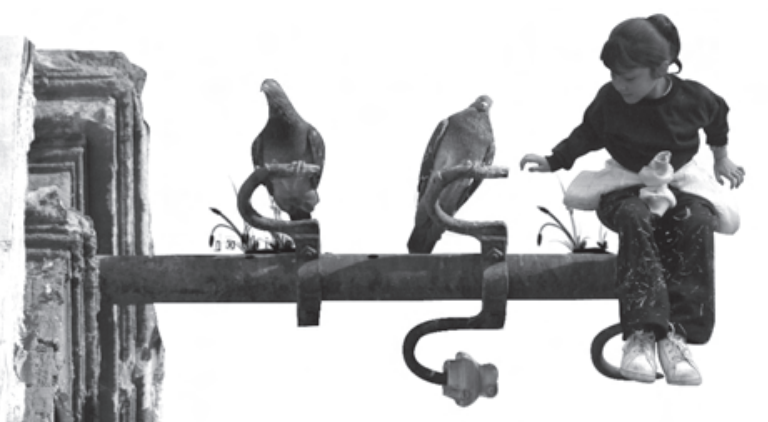

compartidas que generan sentido de pertenencia, facilitan la convivencia urbana, conducen al respeto del patrimonio común y al reconocimiento de los derechos y deberes ciudadanos." 9

No obstante, hablar de lo mínimo compartido conduce a cuestionar cómo un concepto de cultura ciudadana puede considerar entonces la heterogeneidad en la que se desenvuelve la vida en la ciudad, en la cual la distribución de capital cultural, siguiendo a Bourdieu ${ }^{10}$, no se da de la misma forma para todos y cada uno, pues dependiendo de la posición que un sujeto ocupe dentro del espacio social, tendrá un disposición particular y desarrollará una manera de concebir la legalidad, que no necesariamente esté en concordancia con la de los otros.

En concordancia con lo anterior, y desde las experiencias educativas que también se han desarrollado en la ciudad a propósito del tema, el acatamiento y concordancia entre los tres sistemas de regulación -ley, moral y cultura- se rescata como principio de la formación en ciudadanía. Sin embargo, se toman también en consideración las formas particulares de relaciones que establecen las personas en un ámbito urbano, y además, las distintas formas de acción en el ámbito de lo participativo.

En este sentido, el programa Pedagogía Ciudadana concibe la cultura ciudadana partiendo del reconocimiento del sujeto social como agente activo que tiene un sentido sobre lo colectivo, y que este sentido tiene lugar sólo

8 Weber, citado por Geertz, Clifford. La interpretación de las culturas, Gedisa, Barcelona, 1989, p. 20

9 Plan de Gobierno Distrital. Para Formar Ciudad. Bogotá. 1995. Cap. 1. Art. 6. Departamento Administrativo de Planeación Distrital.

10 Bourdieu, Pierre. La distinción. España: Taurus, 1998. 
a través de un proceso social donde se construyen colectivamente prácticas que permiten desarrollar una ciudadanía activa. El programa considera que para que lo compartido sea así, se debe dar un proceso de construcción colectiva en donde la dimensión de la acción sea protagonista en este proceso.

Para la investigación, la cultura ciudadana se entiende como prácticas sociales que generan convivencia, pertenencia urbana, respeto por el patrimonio público, reconocimiento de derechos y deberes y participación; razón por la cual estas cinco categorías se han denominado "componentes de la cultura ciudadana".

Derechos colectivos: estos derechos constituyen un marco importante de acción y referencia para la vida en la ciudad (estos derechos nacen después de la Segunda Guerra Mundial), en la medida en que trascienden los intereses individuales y se ocupan de los intereses del grupo, y porque generalmente posibilitan las condiciones materiales para el desarrollo efectivo de los demás derechos. Así, por ejemplo, el derecho colectivo a un ambiente sano crea las condiciones para garantizar el derecho individual a la vida o a la salud. ${ }^{11}$

En el anterior sentido, los derechos colectivos no son opuestos a los individuales, sino que se complementan y se necesitan unos a otros. De otra parte, en la práctica de los derechos colectivos están inmersos los derechos de otras generaciones, ya que los asuntos aquí tutelados trascienden el tiempo presente e involucran la pervivencia de la especie humana y del planeta mismo.
Con las anteriores premisas, el programa de Pedagogía Ciudadana considera que los derechos colectivos son relevantes para facilitar la formación en Cultura ciudadana, por tanto, a partir de los catorce derechos establecidos en el artículo 4 de la ley 492 de 1993, se establecieron cuatro grandes conjuntos de estos derechos (medio ambiente; espacio público y desarrollo urbano; moralidad administrativa y patrimonio cultural), los cuales constituyen el eje temático esencial de la investigación.

Educación y pedagogía: existen distintas formas de hacer que los ciudadanos conozcan sus derechos y cumplan con sus deberes, sin embargo, la apuesta por la pedagogía surge de la comprensión de la educación como una estrategia de socialización que permite a las personas su desarrollo integral.

En este sentido, el programa de Pedagogía Ciudadana asume que la educación cumple con dos funciones importantes en la formación de las personas. Por una parte, permite a cada uno conocer el grupo al cual pertenece, es decir, apropiarse de su cultura, conocer las maneras en que se espera que se comporte y se haga miembro del grupo social, y de otro lado, la educación permite modificar el contexto; a través de la educación las personas desarrollan su propia interpretación del mundo, y por esa vía inciden en su contexto y producen cambios.

11 Grijalva, A. ¿Qué son los Derechos Colectivos? Disponible en: http://www.uasb.edu.ec/padh/centro/pdf1/GRIJALVA\%20 AGUSTIN.pdf 


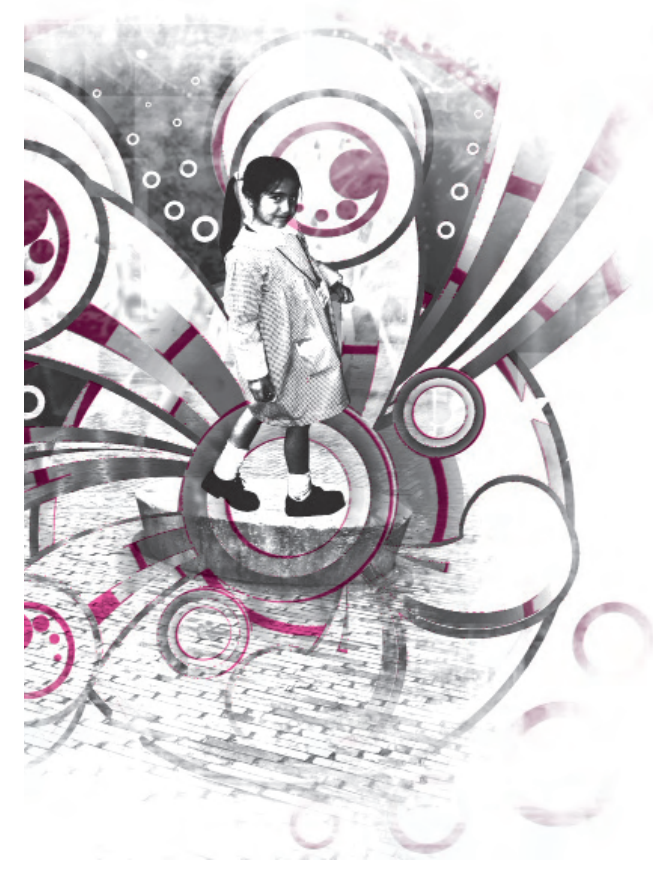

Con base en las premisas y los conceptos anteriores, el programa en Pedagogía Ciudadana persigue, por una parte, la formulación de un proyecto pedagógico para la formación en cultura ciudadana que fomente el sentido de pertenencia, la convivencia urbana, el respeto por el patrimonio común, el reconocimiento de los derechos y deberes ciudadanos, y la participación a través de los Derechos Colectivos, y por otra, la creación de un sistema de indicadores, a partir de una línea de base por ciclos educativos y un modelo estadístico de interpretación de datos, sobre el estado de la cultura ciudadana en la población escolar.

\section{Metodología}

Este apartado resume los principales planteamientos en cuanto a la población objetivo, lineamientos de formación, instrumentos y métodos de recolección de información, y su posterior análisis.

Se debe señalar que existen diferentes tipos de investigación según Zorrilla (1993): básica, aplicada, documental, de campo o mixta ${ }^{12}$. Para efectos prácticos del programa de Pedagogía Ciudadana se describe a continuación la diferencia entre investigación básica y aplicada.

La básica, denominada también pura o fundamental, busca el progreso científico, acrecentar los conocimientos teóricos, sin interesarse directamente en sus posibles aplicaciones o consecuencias prácticas; es más formal, persigue las generalizaciones y el desarrollo de una teoría basada en principios y leyes.

De otro lado, la investigación aplicada guarda íntima relación con la básica, pues depende de los descubrimientos y avances de ésta, y se enriquece con ellos, pero se caracteriza por su interés en la aplicación, utilización y consecuencias prácticas de los conocimientos. La investigación aplicada busca el conocer para hacer, para actuar, para construir y para modificar.

De esta manera, como el interés del programa es intervenir a la población escolar en el tema de Cultura ciudadana, por definición, esta es una investigación de carácter aplicado.

12 Zorrilla. Tipos de investigación. 1993. [Documento en línea] Disponible en: http://.A|/investipos.htm (1 of 4) [Consulta: 2008, Febrero 04.] 
Por otra parte, cuando se habla de una investigación de carácter longitudinal se hace referencia a la posibilidad de trabajar durante un lapso de tiempo determinado con una misma población. Existen dos diferentes formas de estudios longitudinales: los estudios de panel, donde se atiende a la misma muestra a lo largo del tiempo, y los estudios de no panel, que no trabajan con la misma población, pero sí con una muestra con similares características que permite sacar conclusiones.

En síntesis, esta es una investigación longitudinal-aplicada que presenta las siguientes características metodológicas:

Ser interdisciplinar: En el sentido de establecer relaciones constitutivas y complementarias entre varias disciplinas, tanto en el nivel teórico, de construcción de conceptos y categorías, como de modalidades de intervención y análisis. Para ello, el programa cuenta con un grupo de profesionales en distintas áreas del conocimiento que a través de sus experticias realizan el trabajo de complementariedad teórica y práctica del programa. Estos profesionales son de disciplinas como pedagogía, sicología, sociología, artes, comunicación social, arquitectura, antropología y derecho, lo que le proporciona una riqueza a la construcción de la categoría de cultura ciudadana y al proceso formativo en la población de la ciudad.

Ser longitudinal: El Programa parte de la idea de que la construcción de una ciudadanía activa es idónea cuando se inicia desde las primeras etapas de la vida, por esta razón, se trabajará por un período de tiempo con una misma población (estudio de panel), la cual será objeto/ sujeto de la intervención.

En este sentido, la riqueza de un estudio longitudinal radica en que la investigación puede seguir la trayectoria de una misma población y no verse en la necesidad, para su validación, de comparar dos grupos que han sufrido cambios significativos a través del tiempo.

Ser a largo plazo: El programa busca que niños, niñas y jóvenes adquieran herramientas sociales para la vida en común. Por tanto, la formación en cultura ciudadana debe hacerse a través de un proceso que contemple, al mismo tiempo, las características físicas, emocionales, sociales y culturales de la población.

Ser interinstitucional: El programa considera que, para alcanzar una formación en cultura ciudadana que cobije los distintos escenarios en los que se desenvuelven y desenvolverán los niños, niñas y jóvenes en su vida cotidiana, y más adelante en su vida adulta, tanto la comunidad educativa como las entidades relacionadas con el tema, deben trabajar en conjunto y articuladamente para dotar a la ciudad de una propuesta de formación sostenible, que se refleje cuando estos vivan como adultos.

Ser cualitativo y cuantitativo: El programa considera que si bien el proceso de formación en cultura ciudadana está en su mayor parte guiado por un agente formador, el cual puede reconocer, construir y medir variables objetivas de formación, también la valoración que realicen agentes involucrados en el proceso formativo resulta significativo para comprender integralmente la construcción de cultura ciudadana como una experiencia colectiva.

En este sentido, el programa considera viable el uso de métodos e instrumentos cuantitativos y cualitativos en la construcción, análisis e instrumentalización de sus objetivos, dentro de los cuales está la creación de un modelo estadístico de medición de cultura ciudadana en niños, niñas y jóvenes, así como una propuesta de medición de las intervenciones que incluye instrumentos cualitativos. 


\section{Instrumentalización}

\section{i. Población}

En el caso de la investigación aquí planteada, se busca investigar a niños/niñas de ocho años y acompañarlos durante ocho años, para así reportar cambios a nivel cognitivo, afectivo y comportamental en relación con cultura ciudadana, a manera de panel. De esta manera, el programa de Pedagogía Ciudadana proyecta trabajar de manera longitudinal con una muestra representativa de la población escolar del Distrito Capital. Sin embargo, dada la posibilidad de deserción escolar, es probable contar con población de las mismas características de la muestra seleccionada inicialmente, continuando con una población de no panel.

Asimismo, el proyecto atiende a un sistema escolar organizado por ciclos, de acuerdo con la nueva estructura educativa para Bogotá, propuesta por la Secretaría de Educación Distrital, que se encuentra en desarrollo. La actual estructura organizada en grados se modificará en ciclos, correspondiendo el primer ciclo a los grados preescolar, primero y segundo; el segundo ciclo a los grados tercero y cuarto; el tercer ciclo a los grados quinto, sexto y séptimo; el cuarto ciclo a los grados octavo y noveno, y el quinto ciclo a los grados décimo y once.

Dado lo anterior, y considerando las características socio-afectivas de los niños en cada uno de los ciclos, se decidió que la mejor etapa de comienzo de la formación de cultura ciudadana es el ciclo dos. Así, aunque no se

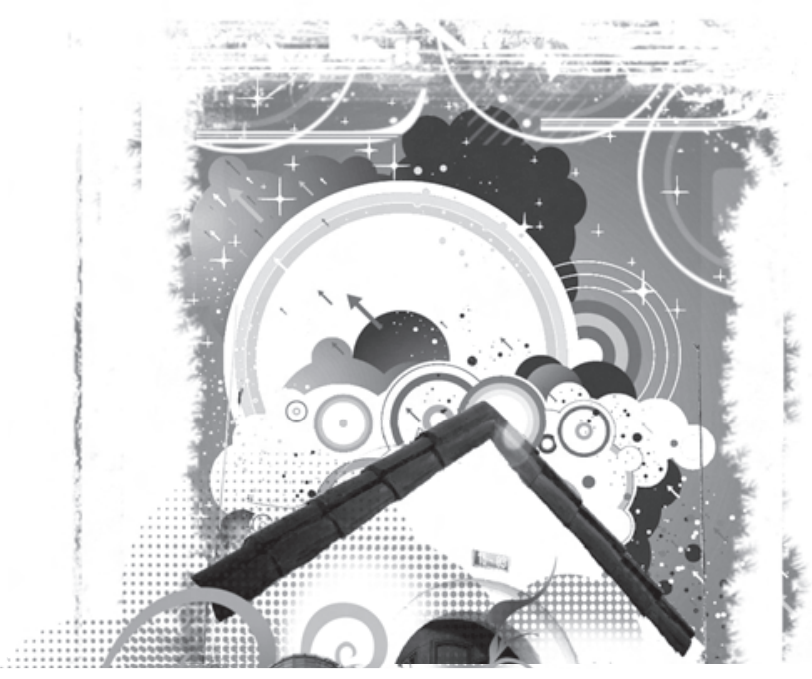

descartan o se desconocen momentos más tempranos de formación y otros espacios propicios para ello (como la familia), se considera que, por el carácter de las intervenciones planteadas, la edad más propicia para esta investigación es entre los 8 y 11 años, de niños/niñas que se encuentran escolarizados en Bogotá.

En este sentido, para elegir la muestra de la investigación se tuvo en cuenta que la unidad de trabajo es la institución escolar y se seleccionaron instituciones educativas que representan las diferentes características de la población en términos cuantitativos, eligiendo al menos un colegio por localidad, y como mínimo un colegio que presenta una de las siguientes características y tipologías: 
- Colegios según tamaño (grandes o pequeños)

- Colegios según creencia religiosa (religiosos o laicos)

- Colegios según género (femeninos, masculinos, mixtos)

- Colegios según calidad (alta, media, baja, según resultados del ICFES)
Del mismo modo, y con el objeto de realizar un análisis global de la ciudad, el programa planteó una división por zonas para analizar las diferencias de acuerdo a la especialización de los colegios. La selección de estas zonas respondió a un criterio puramente administrativo y de gestión del proyecto.

Gráfica 1: Organización por zonas de Bogotá para el Programa de Pedagogía ciudadana.

Gráfica No. 1. Organización por zonas de Bogotá para el programa de Pedagogía Ciudadana
1. Usaquén
2. Chapinero
3. Santa $\mathrm{Fe}$
4. San Cristobal
5. Usme
6. Tunjuelito
7. Bosa
8. Kennedy
9. Fontibón
10. Engativá
11. Suba
12. Barrios Unidos
13. Teusaquillo
14. Mártires
15. Antonio Nariño
16. Puente Aranda
17. Candelaria
18. Rafael Uribe
19. Ciudad Bolívar
20. Sumapaz

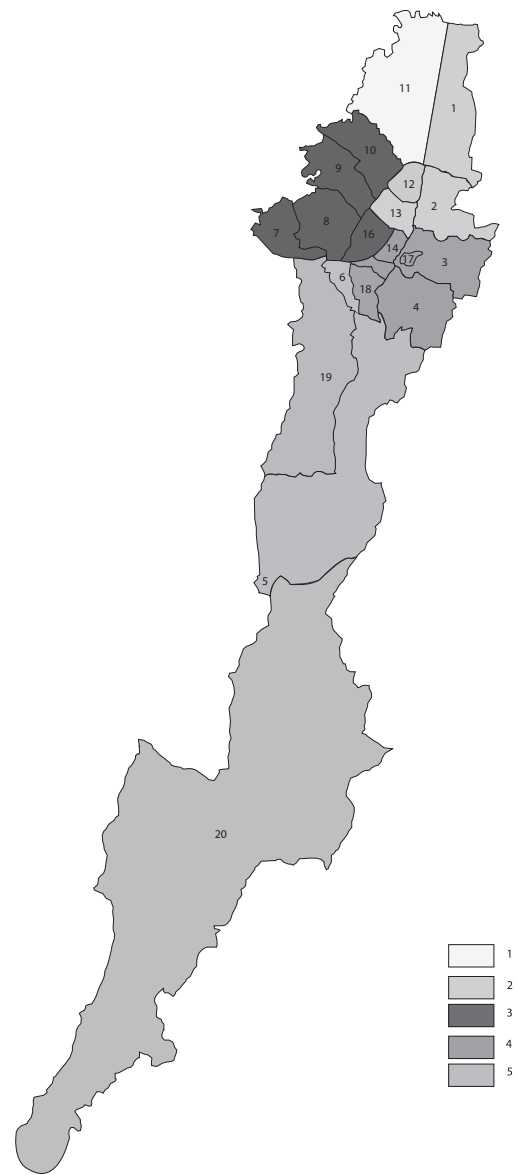




\section{ii. Estructura general del programa}

En este apartado se presentan la estructura lógica y el proceso para adelantar la investigación, los cuales nos permiten observar el enfoque metodológico específico y las actividades de cada proceso.

En primera instancia, resulta necesario señalar que las investigaciones en el IDEP se desarrollan en cinco fases: Identificación, formulación, desarrollo, evaluación y socialización. Para efectos de la presente investigación, la fase de identificación se realizó previamente, y en ella se identificó, de manera general, el problema que es necesario resolver, la justificación de la investigación, los objetivos, las metas, la población y los costos.

La Fase de formulación genera el proyecto definitivo en donde se establecen, de manera concreta y específica, los objetivos, el marco teórico, las hipótesis y la metodología de investigación. Además, determina los procedimientos propios de las etapas subsiguientes: desarrollo, evaluación y socialización.

La fase de desarrollo implementa de manera específica las acciones planteadas en la formulación. Para este caso, se estructura en etapas que corresponden a cada uno de los ciclos según la nueva organización escolar para Bogotá.

De esta forma, la fase inicia con una primera etapa enfocada en ciclo segundo, y continúa, de esta manera, con las demás etapas y ciclos correspondientes. Esta primera etapa está prevista para el año 2009 y 2010, y así sucesivamente hasta terminar.
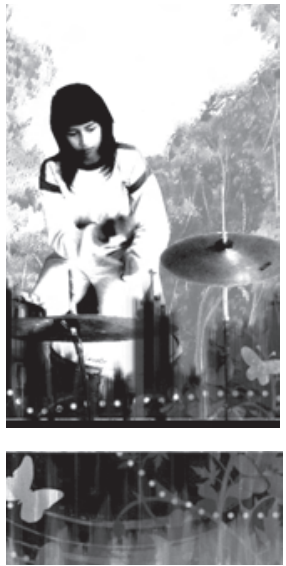

La fase de evaluación se realiza en dos niveles de acuerdo al alcance. Un primer nivel que corresponde a cada una de las etapas de la fase de desarrollo, y consiste en establecer si se cumplieron los objetivos de la investigación mediante la aplicación de unos indicadores previamente establecidos. Un segundo nivel que tiene en cuenta los resultados de las evaluaciones parciales para construir la evaluación general de la investigación. Los resultados de la evaluación sirven para establecer conclusiones y recomendaciones generales de la investigación, y permiten sistematizar la experiencia con el fin de entregarle a la ciudad un programa probado de formación en cultura ciudadana en niños, niñas y jóvenes escolarizados de Bogotá en el 2017.

Finalmente, la fase de socialización pretende dar a conocer los resultados y experiencias del proceso de investigación mediante el desarrollo de actividades e instrumentos de divulgación, con base en los resultados obtenidos. 


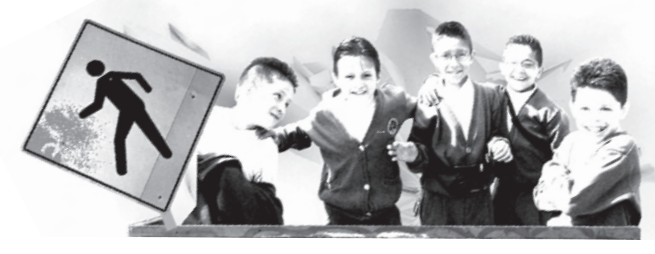

Gráfica No. 2. Estructura general del programa de Pedagogía Ciudadana

\begin{tabular}{|l|c|c|c|c|c|c|c|c|c|c|}
\hline \multicolumn{1}{|c|}{ FASES } & 2008 & 2009 & 2010 & 2011 & 2012 & 2013 & 2014 & 2015 & 2016 & 2017 \\
\hline Identificación & & & & & & & & & & \\
\hline & & & & & & & & & & \\
\hline
\end{tabular}

\section{iii. Procesos de la fase de desarrollo}

Teniendo en cuenta que los objetivos, hipótesis y preguntas de investigación se centran en la aplicación y prueba de un proyecto pedagógico de formación en cultura ciudadana para población escolar, y que este proceso requiere el desarrollo de acciones directas sobre la población, se plantea la necesidad de organizar las actividades de la fase de desarrollo en dos componentes funcionales, un componente técnico y uno administrativo. El primero, enfocado en el proceso académico, y el otro, orientado a la organización administrativa; definición y aplicación de acciones, instrumentos jurídicos y comunicativos, para el manejo y gestión de recursos. De acuerdo con lo anterior, la fase de desarrollo se plantea en dos componentes. 
Gráfica No. 3. Componentes de la fase de desarrollo

COMPONENTE TÉCNICO

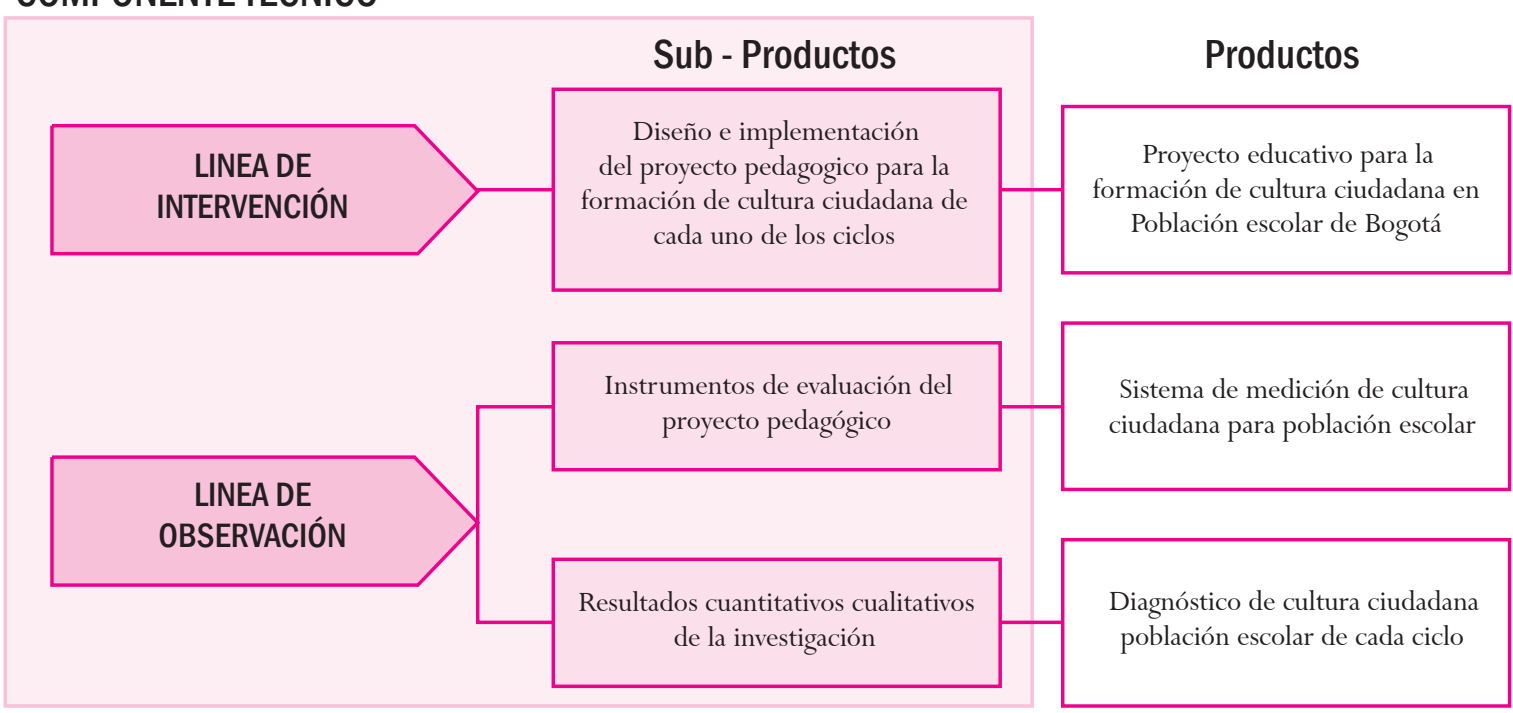

COMPONENTE ADMINISTRATIVO

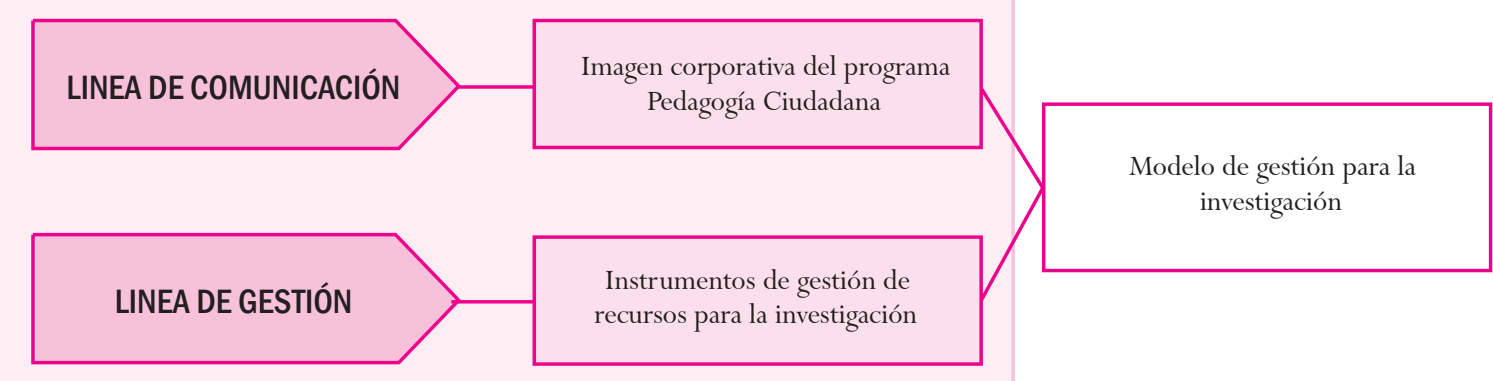

\section{iv. El componente técnico}

Dentro de este componente se llevan a cabo los procesos propios de la investigación, con un enfoque de "investigación longitudinal-aplicada", los cuales han sido estructurados en dos líneas de trabajo: una de intervención y otra de observación. La primera, busca determinar un proyecto pedagógico para la formación en cultura ciudadana a través de la implementación efectiva en colegios públicos y privados de Bogotá, mientras que la segunda, busca crear instrumentos de medición y evaluación para probar si la intervención realizada produce un efecto transformador en la cultura ciudadana y por qué. 
Con el propósito de alcanzar estos dos objetivos, el programa considera importante la participación activa de la comunidad educativa en la formulación de su proyecto educativo, dado que, sólo a través de ella es posible que las acciones que se emprenden vayan en coherencia y concordancia con lo esperado en el ámbito escolar. Así, se plantea que directivos, padres y profesores retroalimenten el diseño de las intervenciones desde mesas de trabajo.

\section{v. Línea de Intervención}

La formación en cultura ciudadana de niños, niñas y jóvenes de la ciudad, requiere un diseño sistemático que permita el cambio en los comportamientos de la población intervenida. Lo anterior, es lo que se conoce en el programa de Pedagogía Ciudadana como línea de intervención pedagógica que, a su vez, contiene tres elementos fundamentales: proyecto pedagógico, red audiovisual y sistema de evaluación.

\section{Proyecto Pedagógico}

La Cultura ciudadana es un tema que no ha sido abordado sistemáticamente en procesos formativos, pues la mayoría de programas relacionados con el tema tratan la formación de ciudadanía, pero no el de cultura ciudadana como tal.

Existen experiencias internacionales, nacionales y locales que vale la pena revisar con el objetivo de identificar el estado actual de la formación en temas relacionados con cultura ciudadana. No existen programas para población escolar que trabajen de manera particular la Cultura ciudadana, pero en cambio, se encuentran muchos que están orientados a la formación de las distintas dimensiones de la cultura ciudadana: convivencia (Aparicio y Lamus, 2003; Osorio, 2006; Arango, 2003; Corrales, 2006), participación (Alterman y Foglino, 2005), Competencias ciudadanas (Chaux, Lleras y Velásquez, 2004), Derechos Humanos (Rodino, 2006; Amnistía internacional tiene en su página WEB una biblioteca de programas desarrollados en Estados Unidos encaminados a la educación en Derechos Humanos: Human rigths education library), ciudadanía (Fundación presencia), medio ambiente (Fernández, 2006; Corrales, 2006). En Bogotá, el caso más relevante es el de Cultura Ciudadana, promovido por el ex alcalde Antanas Mockus, pero que está dirigido, casi exclusivamente, a población adulta; la Fundación Presencia desarrolló un programa dirigido a la formación en cultura ciudadana e involucró algunas escuelas.

De lo anterior, puede colegirse que los dos aspectos innovadores de la investigación en Pedagogía Ciudadana son tres: De una parte, la población, centrada en el universo escolar; en segundo lugar, el trabajo simultáneo en los cinco componentes de la cultura ciudadana, y finalmente, el uso de la pedagogía como estrategia de formación.

En esta medida, el proyecto parte de la premisa de que la educación es una estrategia de socialización que permite a las personas dos resultados fundamentales. El primero, acceder a su propia cultura, es decir, acceder al grupo humano en el que se desarrolla mediante el conocimiento de las prácticas, costumbres, reglas, roles, etc.; por medio de la educación los individuos conocen su grupo, generan pertenencia por el mismo y aprenden las maneras en que se espera que se comporten; esto es, apropian la cultura de su grupo social. El segundo resultado de la educación es que a través de ella, el sujeto 


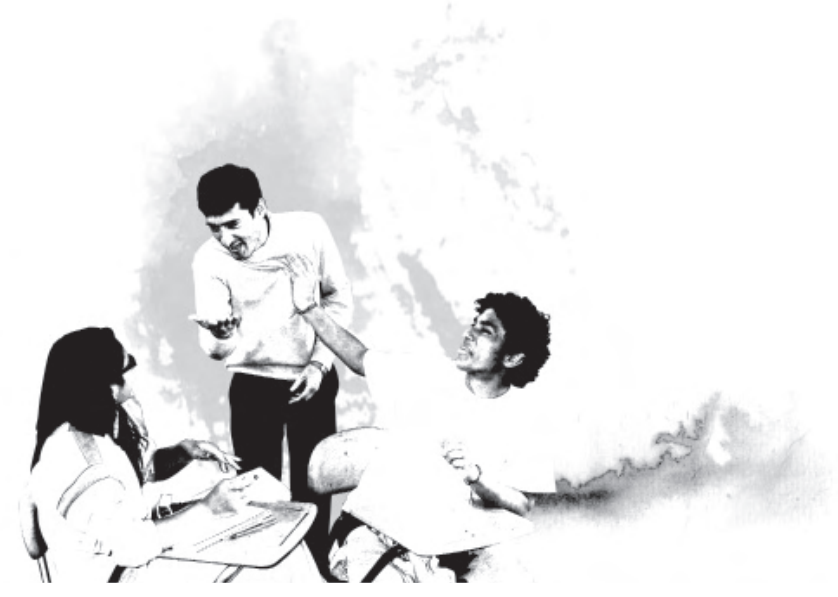

es capaz de desarrollar sus propias capacidades y llegar a impactar su propia cultura, llegando incluso a modificar las prácticas, costumbres y reglas establecidas.

Se comprende, entonces, que la educación es una herramienta de socialización que permite el acceso de los individuos a la cultura, pero que no se limita a adoctrinar a quienes se educan, sino que permite el cambio, el desarrollo de cada persona y promueve tanto la reflexión como la construcción de aportes que mejoran la cultura.

Los procesos pedagógicos, comprendidos como las acciones educativas intencionadas, con objetivos específicos, metodología y una serie de principios pedagógicos, establecen la manera en que se llevan a cabo las acciones educativas, por esta razón, la propuesta del programa Pedagogía Ciudadana consiste en abordar la cultura ciudadana utilizando estrategias pedagógicas que conciben a quien aprende como un sujeto activo, constructor de conocimiento y capaz de modificar su contexto.
La educación, al ser concebida como una herramienta de socialización, carga con la expectativa social de formar a quienes ingresan en el sistema de educación formal en diferentes campos del saber. Se espera que las personas que asisten a la escuela o a cualquier otro espacio de educación formal, evidencien características de dominio de ciertos temas y hagan uso de un conjunto importante de competencias y habilidades. Dentro de este grupo de saberes se encuentran típicamente los conocimientos disciplinares específicos, sin embargo, hace algún tiempo se ha despertado un gran interés por hacer explícita la función de la educación en la tarea de formar personas capaces, más allá de dominar conceptos y teorías, de vivir con sus semejantes.

Este interés se ha plasmado en documentos como el informe UNESCO de la comisión internacional sobre la educación en el siglo XXI, La educación encierra un tesoro, que inicia con palabras de Jacques Delors, el cual señala que "frente a los numerosos desafíos del porvenir, la educación constituye un instrumento 


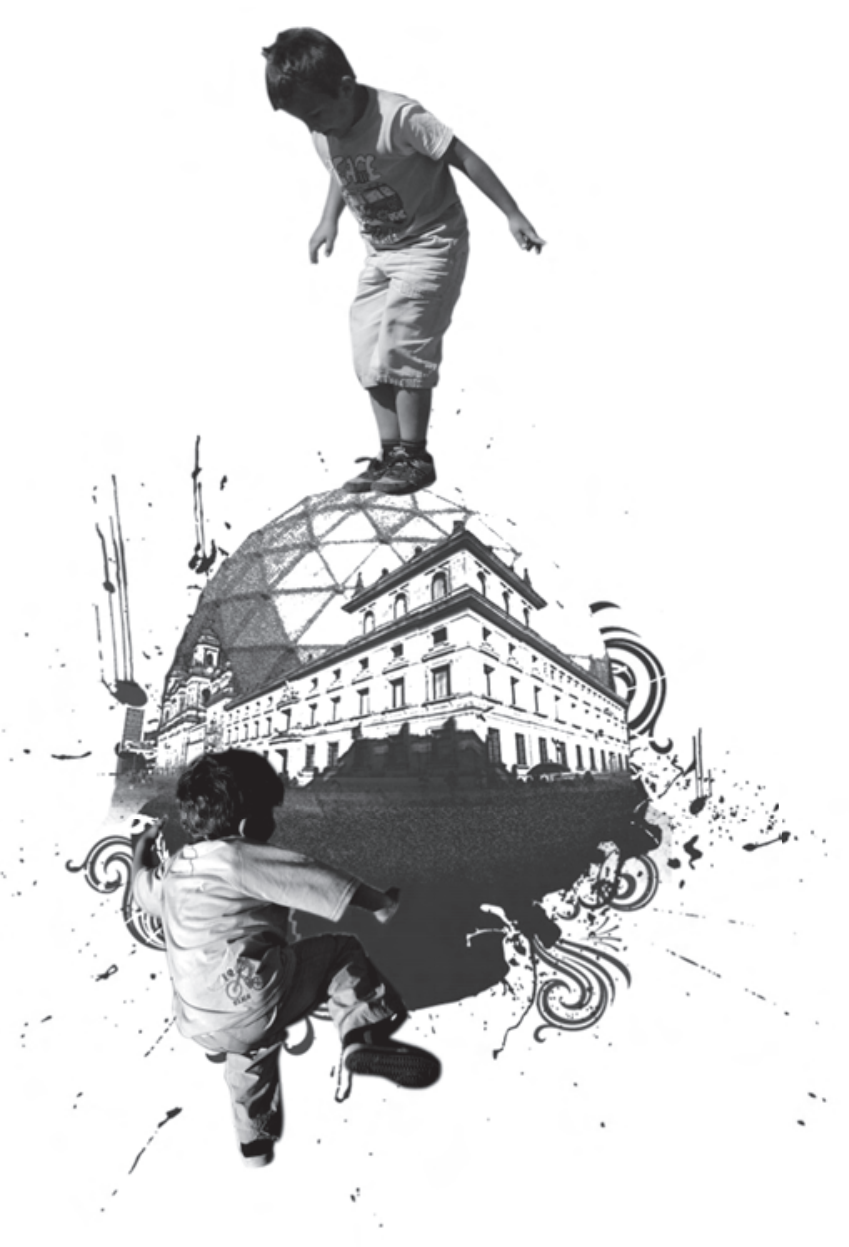

indispensable para que la humanidad pueda progresar hacia los ideales de paz, libertad y justicia social... como una vía, ciertamente entre otras pero más que otras, al servicio de un desarrollo humano más armonioso, más genuino, para hacer retroceder la pobreza, la exclusión, las incomprensiones, las opresiones, las guerras, etc." (Delors, 1996)

Sin lugar a dudas, necesitamos aprender a vivir juntos, por tanto, el informe antes mencionado propone, dentro de los cuatro pilares de la educación para el siglo XXI, el aprender a conocer, aprender a hacer, aprender a vivir juntos y aprender a ser. Estos pilares son fundamentales en el desarrollo ciudadano y justifican que se desarrollen programas como Pedagogía Ciudadana, que se encuentran orientados hacia los principios de organizaciones internacionales como la UNESCO.

La Asamblea General de las Naciones Unidas en el año 1999 realizó la declaración sobre la Cultura de Paz en la que estableció que los años 2001 a 2010 constituyen el Decenio Internacional de una cultura de paz y no violencia para los niños del mundo. En el artículo 1 se conceptualiza la Cultura de Paz como "un conjunto de valores, actitudes, tradiciones, comportamientos y estilos de vida basados en: a) El respeto a la vida, el fin de la violencia, la promoción y la práctica de la no violencia por medio de la educación, el diálogo y la cooperación; b) El respeto pleno de los principios de soberanía, integridad territorial e independencia política de los Estados, y de no injerencia en los asuntos que son esencialmente jurisdicción interna de los mismos, de conformidad con la Carta de las Naciones Unidas y el derecho internacional; c) El respeto pleno y la promoción de todos los derechos humanos y las libertades fundamentales; d) El compromiso con el arreglo pacífico de los conflictos; e) Los esfuerzos para 
satisfacer las necesidades de desarrollo y protección del medio ambiente de las generaciones presentes y futuras; f) El respeto y la promoción del derecho al desarrollo; g) El respeto y el fomento de la igualdad de derechos y oportunidades de mujeres y hombres; h) El respeto y el fomento del derecho de todas las personas a la libertad de expresión, opinión e información; i) La adhesión a los principios de libertad, justicia, democracia, tolerancia, solidaridad, cooperación, pluralismo, diversidad cultural, diálogo y entendimiento; por parte de todos los niveles de la sociedad y entre las naciones.

Por otro lado, el Departamento Nacional de Planeación (DNP) presentó en el año 2006 un documento que evidenciaba los niveles de ciudadanía en Colombia, basándose en datos del observatorio de cultura urbana, alcaldías, instituto de medicina legal y otras entidades nacionales y distritales que manejan este tipo de información. En este documento se presentan resultados de la encuesta realizada en Bogotá, en 2003, con población mayor de 16 años, encontrando que existen altos porcentajes de bogotanos que justifican desobedecer la ley por distintas razones, como lo indica la tabla 3.

\begin{tabular}{l|c}
\hline \multicolumn{1}{c|}{ Indicador } & Bogotá \\
\hline $\begin{array}{l}\text { Porcentaje de personas que justifica desobedecer } \\
\text { la ley cuando es la única manera de alcanzar los } \\
\text { propios objetivos. }\end{array}$ & $19,8 \%$ \\
\hline $\begin{array}{l}\text { Porcentaje de personas que justifica desobedecer la } \\
\text { ley cuando es provechoso económicamente. }\end{array}$ & $6,2 \%$ \\
\hline $\begin{array}{l}\text { Porcentaje de personas que justifica desobedecer la } \\
\text { ley cuando es la única manera de ayudar a la familia. }\end{array}$ & $40,6 \%$ \\
\hline $\begin{array}{l}\text { Porcentaje de personas que justifica desobedecer la } \\
\text { ley cuando es loa costumbrado }\end{array}$ & $7,3 \%$ \\
\hline
\end{tabular}

Tabla 3.Porcentaje de razones para desobedecer la ley. Fuente: Alcaldía Mayor de Bogotá, Instituto Distrital de Cultura y Turismo: Observatorio de Cultura Urbana. Bases de datos de la encuesta de cultura ciudadana aplicada en Bogotá, 2003.
El documento también indica que en el año 2001, el 17\% de los bogotanos no conocía ninguna norma de tránsito vehicular y que en el 2004 hubo 665 accidentes fatales en la ciudad.

Con relación al maltrato hacia los niños, se reporta que en 2004 el 12\% de personas admitió haber golpeado, para castigar, a un niño con un objeto; y se informó que el 63\% de las personas grita a los niños y el 25\% acepta el uso de violencia física como forma de castigarles, adicionalmente, más del 40\% de los bogotanos acepta haber sido agredido durante su infancia.

De otra parte el 10,4\% de personas de Bogotá considera que es mejor tener un arma para protegerse, y en muchos casos no realizan denuncias de los delitos de los que son víctimas por diferentes razones, como se muestra en la tabla 4.

\begin{tabular}{l|c}
\hline & Bogotá \\
\hline $\begin{array}{l}\text { Porcentaje de personas que no denuncia porque las } \\
\text { autoridades no hacen nada. }\end{array}$ & $43,05 \%$ \\
\hline $\begin{array}{l}\text { Porcentaje de personas que no denuncia porque } \\
\text { considera los delitos como ofensas o pérdidas } \\
\text { menores. }\end{array}$ & $19,43 \%$ \\
\hline $\begin{array}{l}\text { Porcentaje de personas que no denuncia porque no } \\
\text { sabían cómo o dónde denunciar. }\end{array}$ & $10,80 \%$ \\
\hline $\begin{array}{l}\text { Porcentaje de personas que no denuncia porque antes } \\
\text { había denunciado y no pasó nada. }\end{array}$ & $3,38 \%$ \\
\hline
\end{tabular}

Tabla 4. Porcentaje de razones por las cuales no se denuncian los delitos. Fuente: DANE, Encuesta de Victimización 2004, presentación electrónica.

Los datos anteriores, evidencian la importancia de desarrollar programas que promuevan la cultura ciudadana, permitiendo a los habitantes de la ciudad modificar sus creencias y comportamientos hacia la ciudad y generando mayor confianza en las entidades del Estado. 
Como se hizo evidente en los antecedentes, en nuestro país y en la ciudad de Bogotá se han desarrollado distintos programas que buscan promover la ciudadanía, la cultura ciudadana y algunos temas que guardan relación con estos conceptos, tanto en los contextos escolares como en espacios comunitarios y virtuales, obteniendo distintos resultados, sin embargo, no existe un abundante cuerpo de investigaciones que haga posible identificar cuáles son las características por las que un programa puede resultar más efectivo para la ciudad de Bogotá.

La mayoría de los programas buscan desarrollar ciertas competencias y modificaciones en los estudiantes, y en general en el contexto, utilizando distintas estrategias y metodologías, no obstante, no suelen estar acompañados de procesos rigurosos de evaluación que permitan establecer su impacto a largo plazo, adicionalmente, las poblaciones son diversas, y en cultura ciudadana como tema global no se ha tenido en cuenta a los estudiantes de educación básica.

Estas razones justifican el diseño, implementación y evaluación de un programa que forme cultura ciudadana en niños y jóvenes de Bogotá, teniendo en cuenta las particularidades de la ciudad, sus necesidades y las oportunidades que ésta ofrece como espacio de formación y desarrollo de la ciudadanía activa.

Por tales motivos, se plantean, a manera de directrices del proyecto pedagógico, los siguientes interrogantes: ¿Existen contenidos que resultan más relevantes que otros para enseñar cultura ciudadana? ¿Qué características debe tener la evaluación idónea para el programa de formación de cultura ciudadana? ¿Cuáles son las particularidades de los procesos de formación en cultura ciudadana según la edad y la etapa de desarrollo de los niños y jóvenes participantes? ¿Cuáles son las características metodológicas de las alternativas pedagógicas que resultan pertinentes para formar a niños, niñas y jóvenes en cultura ciudadana?

De este modo, y con el propósito de atender a las anteriores cuestiones, surge entonces como objetivo general, diseñar, implementar y evaluar una estrategia pedagógica para formar Cultura ciudadana en población escolarizada de Bogotá entre los años 2009 y 2017, que promueva la convivencia urbana, el sentido de pertenencia hacia la ciudad, el reconocimiento y ejercicio de los derechos y deberes, el respeto por el patrimonio común y la participación, a través de los derechos colectivos, el arte y la experiencia directa de la ciudad.

Así mismo, se plantean como objetivos específicos: generar vínculos que contribuyan a superar la brecha económica y social entre niños de la ciudad (Redes); contribuir a la generación de conocimiento compartido sobre temas esenciales para la población que este orientado a generar propósitos comunes; realizar aportes significativos en la formación de un ciudadano activo, así como en la articulación entre escuela y cultura urbana.

Por otra parte, es necesario anotar que, con el fin de alcanzar los anteriores objetivos, el proyecto de formación en cultura ciudadana hunde sus raíces en varias propuestas educativas y pedagógicas que resultan coherentes con sus pretensiones y con las ideas que se tienen del rol de la cultura, la educación y los agentes socializadores como el docente, la familia, el contexto y el mismo estudiante. Por tanto, se atiende a los principios generales del constructivismo, del aprendizaje cooperativo, del aprendizaje significativo, de la pedagogía crítica, y a los principios pedagógicos que se derivan de estas teorías. 
Desde esta perspectiva, el proyecto de formación en Cultura ciudadana será desarrollado durante los años 2009 a 2017 con niños escolarizados de la ciudad de Bogotá. El proyecto busca trabajar durante estos ocho años con los mismos estudiantes, es decir, iniciando en su infancia, en el 2009 cuando se encuentren cursando tercer grado (ciclo 2), y finalizar el proceso de formación en el 2017, en su adolescencia, cuando estén cursando el último grado de educación media (ciclo 5).

Esta particularidad de la población a intervenir obliga a que el proyecto emplee metodologías adecuadas para las diferentes edades y características de los momentos evolutivos de los participantes. Este es un elemento importante en el diseño metodológico, pues no existe una única forma de realizar el proceso formativo, sino que se utilizan diferentes estrategias, teniendo en cuenta las características físicas, cognitivas y sociales de los sujetos.

Adicionalmente, el proyecto se basa en el arte como estrategia que permite desarrollar habilidades sociales e individuales, indispensables para el ejercicio de la ciudadanía activa.

En relación con los espacios en los que se desarrolla el proyecto, es valioso mencionar que se intentan relacionar los espacios escolares tradicionales, es decir, las aulas de las instituciones educativas, con los espacios de la ciudad, para alcanzar experiencias educativas que permitan acceder a la ciudad en sus dimensiones reales y conocer sus condiciones y posibilidades.

Los espacios de aula en la institución educativa son adecuados para trabajar contenidos de manera estructurada, con actividades precisas y objetivos específicos. En el aula los estudiantes aprenden, teorizan,

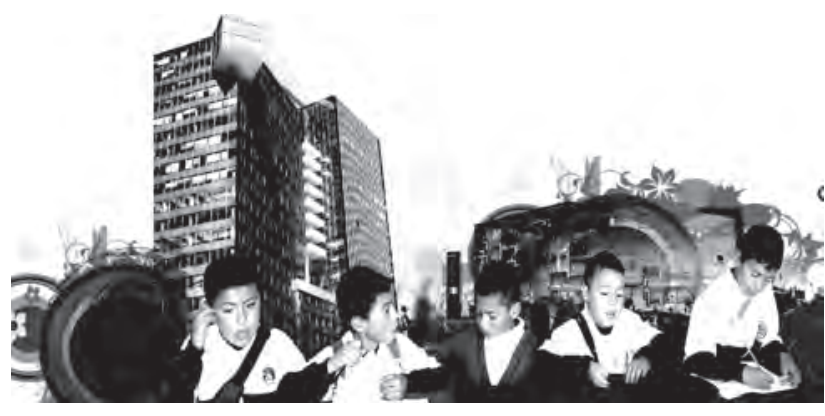

discuten, conciertan y tienen una figura docente que apoya su proceso de construcción de conocimiento. Estos espacios facilitan el seguimiento de los equipos de trabajo y, aunque no se dedican exclusivamente a ello, tienen un énfasis que busca apoyar a los estudiantes con la comprensión cognitiva de los contenidos. Es decir, el aula es un espacio propicio para la estructuración cognitiva y para valorar los alcances de cada niño o joven en su proceso de formación en cultura ciudadana. No obstante, considerando el alto valor de estos espacios, adolecen de la experiencia real de la ciudad. Los niños en el aula están invitados a imaginar su ciudad o a trabajar sobre los conocimientos que ya tienen. Esto resulta limitante, pues la mayoría no reconoce toda la ciudad, por lo que uno de los objetivos del proyecto es que cada niño conozca diferentes zonas y pueda reestructurar su concepto de la misma, en la medida en que aprende sobre ella, pero este aprendizaje requiere de experiencias en la ciudad y no sólo del trabajo con imaginarios de lo que cada uno cree que ésta es.

Otro aspecto metodológico fundamental es el trabajo en proyectos, lo cual resulta coherente con los elementos citados en el marco teórico, en lo que respecta a la pedagogía de proyectos y al aprendizaje cooperativo. 


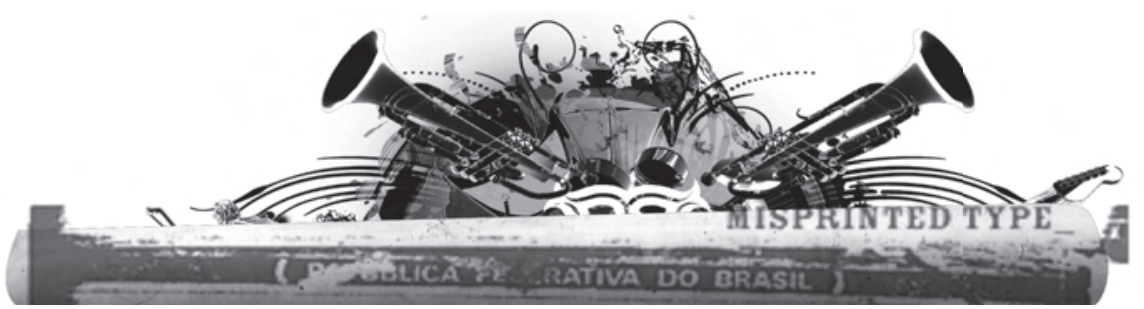

Durante el proceso de formación, los estudiantes constituyen equipos de trabajo para desarrollar diferentes proyectos orientados a resolver problemáticas que los mismos niños identifican en la ciudad; para esto cuentan con el apoyo de los pedagogos. Los espacios de aula y las salidas a la ciudad aportan a los proyectos que cada equipo esté desarrollando, y los pedagogos tienen como misión apoyar a los equipos en el desarrollo de cada proyecto.

Al finalizar cada etapa (grado académico) del proceso de formación en cultura ciudadana los equipos presentan sus proyectos. Por lo demás, existe un aspecto valioso en estos proyectos relacionado con el arte, y es que cada equipo debe construir un video en el que se evidencie su proceso de desarrollo del proyecto. Este video es construido a lo largo de cada etapa de formación por el equipo de niños o jóvenes y un profesional en audiovisuales que los asesora en el guión, en el diseño y ejecución del video. Además, consiste en una estrategia que vincula elementos artísticos con los contenidos del proceso de formación.

Asimismo, el proyecto cuenta con una red virtual de la que hacen parte todas las instituciones educativas vinculadas al proyecto. Esta red constituye un espacio virtual en el que todas las personas vinculadas al proyecto aprenden de los proyectos desarrollados en otras instituciones y a través de ella se pueden generar vínculos institucionales y sociales que promueven el trabajo en equipos interinstitucionales.
Por su parte, la evaluación en el proceso de formación es considerada como una posibilidad de retroalimentación para cada participante, por lo que se realizan evaluaciones de los diferentes componentes del proceso formativo. Se evalúa a los estudiantes para identificar sus características afectivas, cognitivas y comportamentales frente a la ciudad, con el objeto de retroalimentar su proceso. Así, cada estudiante conoce sus resultados y tiene la oportunidad de discutirlos y enriquecerse. Se evalúa a los pedagogos para retroalimentar su trabajo y tener la posibilidad de mejorar sus prácticas con los jóvenes. Se evalúa a los profesionales en audiovisuales para retroalimentar su trabajo y reconocer qué aspectos han resultado más valiosos para los estudiantes en la construcción del video y de la red virtual. Se evalúa la metodología del proyecto, es decir, los recorridos, los espacios de aula y el trabajo en proyectos, del mismo modo, el video y la red se evalúan para determinar el éxito de cada estrategia y los elementos a mantener y mejorar.

\section{Red Pedagógica Audiovisual}

La Red Pedagógica Audiovisual se proyecta como un espacio virtual para el intercambio e interacción de niños, niñas, jóvenes, padres de familia, profesores y comunidad académica vinculada al proyecto. Este mecanismo pretende disminuir barreras físicas y distancias sociales, creando un espacio que fomenta la convivencia ciudadana y promueve el encuentro de personas que de otra manera no pueden llegar a conocerse. Finalmente, esta red es 


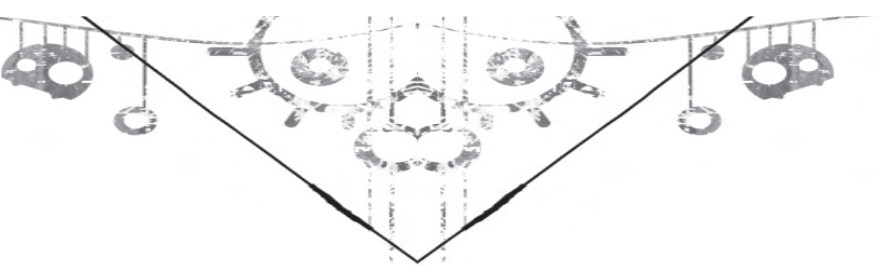

una herramienta de investigación para el programa Pedagogía Ciudadana que permite reconocer los avances de su investigación.

La producción de piezas audiovisuales participativas permite sistematizar los discursos y testimonios de niñas, niños y jóvenes; esta sistematización facilita la visualización de los avances del proceso pedagógico a lo largo del tiempo de intervención. Las piezas audiovisuales ofrecen la posibilidad de crear documentos que testifiquen lo aprendido sobre cultura ciudadana.

\section{Sistema de Medición y Evaluación}

La línea de observación pretende definir los instrumentos de medición y evaluación del proyecto pedagógico propuesto en la línea de intervención. El diseño cuenta con dos estrategias de medición que se retroalimentan continuamente. Específicamente, uno de los instrumentos sirve para medir la cultura ciudadana en cada uno de los ciclos y construir la línea de base para el proyecto de investigación que se elabora a partir de la aplicación de dicho instrumento a una muestra representativa de la población escolar de cada ciclo. Dicha línea de base establece las disposiciones ciudadanas (antes de las intervenciones) de los niños y niñas de la ciudad en cada ciclo, constituyéndose en un estudio transversal de dicha población. Esta medición se repite cada dos años a una muestra de niños y niñas seleccionada utilizando la misma metodología de la línea de base, de manera que a partir de múltiples estudios transversales $(2009,2011,2013,2015)$ se pueda construir un punto de referencia para medir el avance en cultura ciudadana.

El instrumento se construyó realizando una matriz que cruza los elementos de la cultura ciudadana, los derechos colectivos y las dimensiones del ser humano, así:

\begin{tabular}{|c|c|c|c|c|}
\hline $\begin{array}{l}\text { Dimensiones de la } \\
\text { cultura ciudadana }\end{array}$ & Convivencia & Participación & Deberes y Derechos & $\begin{array}{c}\text { Respeto por el } \\
\text { Patrimonio Común }\end{array}$ \\
\hline \multicolumn{5}{|l|}{ Derechos colectivos } \\
\hline Medio Ambiente & \multirow{3}{*}{\multicolumn{4}{|c|}{$\begin{array}{c}\text { Cognitivo } \\
\text { Afectivo } \\
\text { Comportamental/ acción }\end{array}$}} \\
\hline Patrimonio Cultural & & & & \\
\hline Espacio Público & & & & \\
\hline
\end{tabular}

Este instrumento es aplicado a una muestra de estudiantes de Bogotá en muestreo bietápico estratificado. La primera etapa consiste en la selección de colegios, y la segunda en la selección de estudiantes. La selección de colegios se realiza teniendo en cuenta dos criterios: naturaleza 
del colegio (oficial o privado) y tamaño; se atiende de manera exclusiva a seis tamaños, tomando como base los siguientes criterios:

1. de 0 a 200 estudiantes

2. de 201 a 600 estudiantes

3. de 601 a 1.000 estudiantes

4. de 1.001 a 2.000 estudiantes

5. de 2.001 a 3.000 estudiantes

6. de 3.001 a 6.000 estudiantes

El tamaño de la muestra es de 3.417 estudiantes y tiene representatividad por tamaño y por naturaleza del colegio. Esta muestra fue determinada con un nivel de confianza del 95\% y un error estimado del 5\%, con afijación proporcional al tamaño del estrato.

Por su parte, el sistema de evaluación del Programa de Pedagogía ciudadana se realiza teniendo en cuenta dos elementos: los resultados y el proceso. Igualmente, se propone realizar una evaluación de carácter individual y otra de carácter general. La primera está orientada a recoger datos que permitan establecer el proceso de cada estudiante en la formación en cultura ciudadana. La segunda pretende evaluar cómo ha sido el proceso de formación en general; cómo se ha desarrollado el proceso de formación en un grupo, en un colegio, en una zona, etc.

En lo que respecta a la evaluación individual cabe anotar que se realiza a los 1395 estudiantes y que los instrumentos de medición de esta evaluación son diseñados por el grupo de pedagogos, quienes establecen el método, así como los formatos correspondientes y las modalidades de aplicación para tener un reporte de cada niño, niña o joven sobre su proceso de formación y, de este modo, cada estudiante cuenta con un indicador de desempeño. No obstante, la interpretación de la evaluación individual puede estar acompañada por otras modalidades de evaluación como el análisis de los proyectos finales, las apreciaciones del pedagogo o el análisis de los grupos, las cuales dependen de los criterios que el grupo de pedagogos construye.

De otro lado, la evaluación general está basada en un método cuantitativo, en el cual se recurre al uso de variables objetivas, medibles y verificables, y en un método cualitativo que recurre a la percepción que tienen los propios participantes del proceso de formación. Esta evaluación realiza un muestreo de la población intervenida (1395 niños y niñas) para realizar el seguimiento. Con base en los principios de la ley de los grandes números, la selección de la población evaluada cubre dentro del conjunto de la población intervenida el $10 \%$ de representatividad (200 niños y niñas) y acude a la comparación de los resultados generales con grupos de referencia (64 niños y niñas) no expuestos a la intervención, con el propósito de identificar las diferencias en la dimensión afectiva, cognitiva y comportamental que permiten medir los resultados del desarrollo del proyecto pedagógico.

Por último, se debe señalar que una vez adelantadas todas las etapas de la investigación, se realizarán las recomendaciones correspondientes, tanto a las instituciones escolares públicas como privadas, para que tomen en cuenta el proyecto pedagógico que se ha monitoreado previamente, y de este modo lograr que la experiencia investigativa trascienda las fronteras de lo experimental, y sea asumida como una posibilidad real en la formación en cultura ciudadana de niños, niñas y jóvenes escolarizados de la ciudad. 


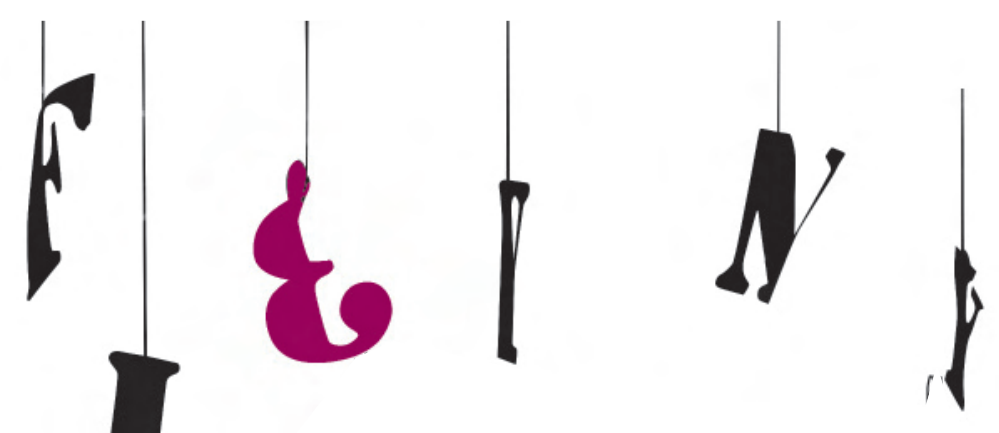

(Disponible en: http://www.fund-culturadepaz.org/DECLARACIONES\%20RESOLUCIONES/Declaracion\%20Cdpaz\%20 Esp.pdf)

Plan de Gobierno Distrital. Para Formar Ciudad, Bogotá, Departamento Administrativo de Planeación Distrital, 1995.

Rodino, A. (Coordinadora). Propuesta curricular y metodológica para la incorporación de la educación en derechos humanos en la educación formal de niños y niñas entre 10 y 14 años de edad. Instituto interamericano de derechos humanos. Unidad pedagógica, 2006.

Taylor, E. Anthropology: An Introduction to the Study of Man and civilization. New York, D. Appleton, 1909.

Touraine, A. El regreso del actor. Buenos Aires, Eudeba, 1987.

Zorrilla. Tipos de investigación, 1993. (Disponible en: http://.A|/investipos.htm) [Consulta: 2008, Febrero 04]. 